\title{
TWO PROBLEMS OF MIXED TYPE FOR THE DAMPED WAVE EQUATION*
}

\author{
BY \\ D. G. BOURGIN \\ University of Illinois
}

This paper is primarily concerned with a mixed problem of somewhat unconventional form for the one dimensional damped wave equation. Although the treatment given is rigorous, the main interest of this work lies in the explicit determination of the solution in closed form and in the methods used. The method of attack pivots on the use of images (or co-images) associated with a hyperbolic metric. The utility of such extensions of the method of images, so useful for equations of elliptic type, was first pointed out by Volterra. ${ }^{1}$ However, the literature contains scant references to the application ${ }^{2}$ and development of this idea. For the purpose of cataloguing the nature of our addition to the class of explicitly soluble problems it may be pointed out that the fundamental integral equations for the main problem are not of convolution type (cf. Sec. 7).

The main problem may be given significant physical interpretation in various fields. For instance, it covers the vibration of a damped string whose length varies linearly with time. One end moves in a vertical slot and the other is coupled to a driving mechanism through an inertialess damped spring. Alternatively we may consider one dimensional gas motion (or sound propagation) in a cylinder of large radius. One end is closed. The other moves at constant rate and is a friction damped elastic diaphragm driven by an exterior mechanism. We may think of our problem as that of a telegrapher's equation problem for an electric cable of steadily increasing length. A fourth application is to problems connected with Schuster's theory of the radiation field in a moving radiating atmosphere in the formulation given by Chandrasekhar. Indeed the writer's interest in this investigation was aroused by a manuscript which Dr. Chandrasekhar communicated to him. ${ }^{3}$ The second problem treated in this article is already a generalization of that of Chandrasekhar's, though the developments are simpler than those for our main problem.

1. Preliminaries. We shall be interested in a boundary value problem associated with

$$
z_{t t}-z_{x x}+z=L(z)=0 .
$$

Before we state it, it seems desirable to indicate in brief the basic theory that we draw on.

Let $z$ and $u$ be solutions of (1.0), then for a continuous rectifiable simple closed curve, $\Gamma$, bounding a domain $S$ we have

*Received Sept. 22, 1947.

'V. Volterra, Leçons sur l'intégration des équations différentielles aux dérivées partielles, A. Herman et Fils; Paris, 1912.

2In the thesis just completed by Mr. A. Charnes (University of Illinois) this method is of central importance in analyzing the influence of a polygonal section type of fuselage on the wing potential for the linearized supersonic flow.

${ }^{3}$ His interesting method has since been published: S. Chandrasekhar, $A$ new type of boundary value problem in hyperbolic equations, Proc. Cambridge Philos. Soc. 42, 250-260 (1946). Actually, the generalization of Chandrasekhar's boundary conditions to problem 2 would give somewhat different conditions than those used in this paper, but these are just as easily handled. On this point $c f$. footnote 4. 


$$
0=\iint_{S}[u L(z)-z L(u)] d S=\int_{\Gamma}\left[u\left(z_{x} t_{s}+z_{t} x_{s}\right)-z\left(u_{x} t_{s}+u_{t} x_{s}\right)\right] d s,
$$

for a clockwise sense of description of $\Gamma$. Besides the Euclidean metric used for $d s$ we associate a hyperbolic metric with (1.01), namely

$$
d \sigma^{2}=d t^{2}-d x^{2} .
$$

The characteristics of (1.0) are the null lines for $d \sigma$, namely $t+x=C, t-x=C$. We shall refer to the members of these two families as plus characteristics and minus characteristics respectively. A pair of vectors orthogonal with respect to this metric are said to be co-normal. Thus the vector $\delta x$, $\delta t$ is co-normal to the tangent vector (to г) $d x, d t$ provided

$$
\delta t d t-\delta x d x=0 .
$$

We denote the co-normal direction by $\nu$. That is to say, we write

$$
\frac{\delta x}{\delta \nu}=\frac{d t}{d s}, \quad \frac{\delta t}{\delta v}=\frac{d x}{d s}
$$

If $\Gamma$ is given by $f(x, t)=0$ then of course

$$
\begin{aligned}
& \frac{d x}{d s}= \pm f_{t} /\left(f_{x}^{2}+f_{t}^{2}\right)^{1 / 2}=\frac{\partial t}{\partial \nu} \\
& \frac{d t}{d s}=\mp f_{x} /\left(f_{x}^{2}+f_{t}^{2}\right)^{1 / 2}=\frac{\partial x}{\partial \nu} .
\end{aligned}
$$

The sign choice is determined by the order on the curve. Thus (1.01) becomes

$$
\int_{\mathrm{r}}\left(v \frac{\partial z}{\partial \nu}-z \frac{\partial v}{\partial \nu}\right) d s=0
$$

We shall take for $v$ the Riemann function

$$
u\left(x, t ; x_{1}, t_{1}\right)=J_{0}\left(\left(t-t_{1}\right)^{2}-\left(x-x_{1}\right)^{2}\right)^{1 / 2} .
$$

For our purpose the significant properties of $u$ are first that in the sector expanding downwards bounded by $t \pm x=t_{1} \pm x_{1}$, it satisfies (1.0) and second that $u$ has the constant value 1 on the two sides of this sector. It is then clear that the tangential derivative of $u$ along these sides vanishes. On the plus characteristic $t+x=C$ we have from (1.03) that

$$
\left(\frac{d x}{d s}, \frac{d t}{d s}\right)=(1,-1)=\left(-\frac{\partial x}{\partial \nu}, \frac{\partial t}{\partial \nu}\right) .
$$

That is to say, for these characteristics

$$
\frac{\partial}{\partial \nu}=-\frac{d}{d s}
$$

On the other hand (1.03) shows that for the minus characteristic $t-x=C$ we get

$$
\frac{\partial}{\partial \nu}=\frac{d}{d s} \text {. }
$$


Hence if $\Gamma$ includes a segment of a characteristic we have as the contribution to (1.04), with $s_{2}>s_{1}$,

$$
\int_{02}^{s .} \frac{\partial z}{\partial \nu} d s= \pm\left. z\right|_{01} ^{\cdot 0}
$$

according as the characteristic is a minus or a plus one.

Our concern is with the solution of (1.0) restricted by boundary conditions on $t=0, P: t+\lambda x=0$ and a line $P^{\prime}$ which is $x=l$ for our main problem and $t+\lambda x=$ $\lambda l$ for our second problem. In view of the linearity of our system we can express the general solution as a sum of three solutions each determined by 0 data on two of the boundaries. Thus consider the solution of (1.0) subject to ${ }^{4}$

$$
\begin{aligned}
& (A): z_{\nu}=\gamma(x, t) \text { on } P^{\prime}, \\
& (B): z=A(x), z_{t}=B(x) \text { on } t=0, \\
& (C): z_{\nu}=h z(x, t)+\psi(x, t) \text { on } P .
\end{aligned}
$$

Let $z^{4}$ be the solution of (1.0) subject to vanishing Cauchy data on $t=0, z_{\nu}=0$ on $P$ and $z_{v}=\gamma(x, t)$ on $P^{\prime}$. Let $z^{B}$ be the solution of (1.0) subject to $z=A(x), z_{t}=B(x)$ on $t=0 ; z_{\nu}=0$ on $P^{\prime}$ and $z_{\nu}=0$ on $P$. Let $z^{c}$ be the solution of (1.0) under the conditions $z_{v}=0$ on $P^{\prime}$, vanishing Cauchy conditions on $t=0$ and $z_{\nu}=h z(x, t)+\psi(x, t)+$ $h\left(z^{A}+z^{B}\right)$ on $P$. It is easily verified that the solution of (1.0) restricted by $(A),(B)$ and $(C)$ is then given by $z=z^{A}+z^{B}+z^{C}$.

It is clear that the crux of the general problem is the determination of $z^{c}$ for $z^{A}$ and $z^{B}$ can be determined by a simpler form of the methods of this paper (or otherwise). Thus we shall concern ourselves only with the solution of (1.0) subject to

$$
\begin{aligned}
& z_{\nu}=0 \text { on } P^{\prime}, \\
& z=z_{t}=0 \text { on } t=0, \\
& z_{\nu}=h z(x, t)+\psi(x, t) \text { on } P .
\end{aligned}
$$

We make the further assumptions that $\psi(0,0)=0$ and that on $P, d / d s \psi(0,0)=0$ $\operatorname{and}^{5} \psi(x, t)_{\varepsilon} C^{\prime}$. We suppose also that $\lambda>1$ and $h \geq 0$ though this last restriction is not significant for our analysis.

2. Geometric considerations. On $P$ consider $s$ increasing with $t$. This will be the situation in our developments. Then $d x / d s$ is negative and $d t / d s$ is positive. From (1.03) we get

$$
\begin{aligned}
& \frac{\partial x}{\partial \nu}=\lambda /\left(\lambda^{2}+1\right)^{1 / 2} \\
& \frac{\partial t}{\partial \nu}=-\left(\lambda^{2}+1\right)^{-1 / 2}
\end{aligned}
$$

\footnotetext{
${ }^{4}$ Chandrasekhar's problem corresponds to the case $\lambda=\infty$. His conditions would amount to changing $h z$ to $z_{s}$ in (C) and $z$, to $z$ in (A). The last modification is evidently trivial for the corresponding $z$ is just as easily found. The first change has the effect of replacing $h$ by $p$ in all the transforms arising in Problem 2. Since, however, the inverse transforms for this case are direct and essentially on record, Cf. 8 , the problem is actually simpler than before.

${ }^{5} C^{n}$ denotes the class of functions continuous up to and including the $n$th derivatives with respect to the independent variables (here a single one).
} 
The key of our method is the use of images. The reader will find a figure helpful. Consider the image in $P$ of the point $\left(x_{0}, t_{0}\right)$ with respect to the hyperbolic metric (1.02). Denote this image by $L$. Then the hyperbolic distances from any point of $P$ to $L$ and to $\left(x_{0}, t_{0}\right)$ are equal. Thus if $L$ is $\left(x^{\prime}, t^{\prime}\right)$ then for all $x$,

whence

$$
\left(\lambda x+t_{0}\right)^{2}-\left(x-x_{0}\right)^{2}=\left(\lambda x+t^{\prime}\right)^{2}-\left(x-x^{\prime}\right)^{2},
$$

$$
\begin{aligned}
x^{\prime} & =-\left(2 \lambda t_{0}+\left(\lambda^{2}+1\right) x_{0}\right) /\left(\lambda^{2}+1\right), \\
t^{\prime} & =\left(2 \lambda x_{0}+\left(\lambda^{2}+1\right) x_{0}\right) /\left(\lambda^{2}-1\right) .
\end{aligned}
$$

It is convenient to use the representation

Thus

$$
\begin{aligned}
& \cosh \alpha=\left(\lambda^{2}+1\right) /\left(\lambda^{2}-1\right), \\
& \sinh \alpha=2 \lambda / \lambda^{2}-1
\end{aligned}
$$

$$
\begin{aligned}
x^{\prime} & =-\left(x_{0} \cosh \alpha+t_{0} \sinh \alpha\right), \\
t^{\prime} & =x_{0} \sinh \alpha+t_{0} \cosh \alpha .
\end{aligned}
$$

We denote the image of $x_{0}, t_{0}$ in $P^{\prime}$ by $R$. The calculations of this section refer to the main problem. Thus

$$
R:\left(2 l-x_{0}, t_{0}\right) .
$$

The image of $L$ in $P^{\prime}$ is denoted by $L R$. That of $R$ in $P$ by $R L$, etc. Hence the point $R L$ has coordinates

$$
\begin{aligned}
x^{\prime} & =2 l+x_{0} \cosh \alpha+t_{0} \sinh \alpha, \\
t^{\prime} & =\quad x_{0} \sinh \alpha+t_{0} \cosh \alpha .
\end{aligned}
$$

It is plain that $\cdots R$ and the image corresponding to this symbol with an added $L$ on the right, namely $\cdots R L$, have equal hyperbolic distances from any point on $P$. A similar remark holds for $\cdots L$ and $\cdots L R$ with respect to $P^{\prime}$. Accordingly the function in (1.05) with $x_{1}, t_{1}$ corresponding to $\cdots R$ and that with $x_{1}, t_{1}$ corresponding to $\cdots$ $R L$ have equal values on $P$. Moreover, the derivatives with respect to $\nu$ of these two $u^{\prime}$ s are equal, but of opposite sign on $P$. Thus with $\left(t-t_{0}\right)^{2}-\left(x-x_{0}\right)^{2}=\left(t-t_{1}\right)^{2}-$ $\left(x-x_{1}\right)^{2}=\mu^{2}$ for $x, t$ on $P$ we have

$$
\left(\lambda^{2}+1\right)^{1 / 2} \frac{\partial}{\partial \nu} J_{0}\left[\left(t-t_{0}\right)^{2}-\left(x-x_{0}\right)^{2}\right]^{1 / 2}=\frac{1}{\mu} \frac{d}{d \mu}\left(J_{0}(\mu)\right)\left(\lambda x_{0}+t_{0}\right)
$$

We have therefore merely to show

$$
\lambda x_{0}+t_{0}=-\left(\lambda x_{1}+t_{1}\right)
$$

for $L: x_{1}, t_{1}$. However, this is an immediate consequence of (2.03).

Denote by $J$ the combination $L R$ and by $J^{n}$ the $n$th iterate. Denote by $K^{n+1}$ the point $K J^{n}$, where $K=R J^{0}=R$. Let $\mathbf{M}$ be the matrix

$$
\left(\begin{array}{cc}
\cosh \alpha & \sinh \alpha \\
\sinh \alpha & \cosh \alpha
\end{array}\right) .
$$


We may give $(a, b)$ the dual interpretation of a point (or vector) and a one rowed matrix. Thus with the familiar algorithm for matrix multiplication, we may write (2.04) as

$$
J:\left(x_{1} t_{1}\right)=\left(x_{0} t_{0}\right) \mathbf{M}+(2 l 0) .
$$

Suppose first $l=0$. Then the coordinates of $J^{n}$ are given by

$$
\left(x_{n} t_{n}\right)=\left(x_{0} t_{0}\right) \mathbf{M}^{n} .
$$

Since $\mathbf{M}$ induces a rotation of angle $\alpha$ in the sense of the hyperbolic metric, i.e. a Lorentz rotation, it follows at once that

$$
\mathbf{M}^{n}=\left(\begin{array}{ll}
\cosh n \alpha & \sinh \cdot n \alpha \\
\sinh n \alpha & \cosh n \alpha
\end{array}\right)
$$

A direct proof of (2.06) proceeds by induction, using the addition laws for the hyperbolic functions.

The contribution of the $l$ terms may be obtained by assuming $x_{0}=t_{0}=0$ and $l \neq 0$. Let $l_{n}, l_{n}^{\prime}$ be the $l$ terms in the $x$ and $t$ coordinates of $J^{n}$. Then according to (2.03) and (2.04)

$$
\begin{aligned}
& l_{n+1}=2 l+l_{n} \cosh \alpha \\
& l_{n+1}^{\prime}=l_{n}^{\prime} \sinh \alpha
\end{aligned}
$$

That is to say

$$
\left(l_{n+1} l_{n+1}^{\prime}\right)=(2 l 0)+\left(l_{n} l_{n}^{\prime}\right) \mathbf{M}
$$

Hence

$$
\left(l_{n} l_{n}^{\prime}\right)=(2 l 0) \sum_{0}^{n-1} \mathbf{M}^{i}=(2 l 0)\left(\begin{array}{c}
e_{n} 0 \\
00_{n}
\end{array}\right)
$$

where $e_{n}$ and $0_{n}$ are the even and odd parts of $\sum_{0}^{n-1} \mathbf{M}^{j}$ and are equal to $\sum_{0}^{n-1} \cosh j \alpha$ and $\sum_{0}^{n-1} \sinh j \alpha$ respectively. Hence $J^{n}: X_{n}, T_{n}$ is given by

$$
\begin{aligned}
& X_{n}=e_{n} l+x_{0} \cosh n \alpha+t_{0} \sinh n \alpha, \\
& T_{n}=0_{n} l+x_{0} \sinh n \alpha+t_{0} \cosh n \alpha .
\end{aligned}
$$

The coordinates of $K^{n}$ are designated by $X_{n}^{\prime}, T_{n}^{\prime}$. It will be clear that $K^{n}$ arises from $J^{n-1}$ by the substitution of $2 l-x_{0}$ for $x_{0}$. We observe that $\cosh j \alpha$ and $\sinh j \alpha$ are even and odd functions respectively as regards both $\alpha$ and $\lambda$. Accordingly the reader may verify the following formulae convenient for our purpose

$$
\begin{aligned}
& e_{n}=\lambda \sinh n \alpha+(1-\cosh n \alpha), \\
& 0_{n}=\lambda(\cosh n \alpha-1)-\sinh n \alpha .
\end{aligned}
$$

It is convenient to change from the coordinate system $(x, t)$ to another, $(w, v)$, in which the coordinate lines are again conormal, i.e. orthogonal in the metric of (1.02). 
We require that $v=0$ give $P$ and that the transformation from $(x, t)$ to $(w, v)$ preserve the hyperbolic distance. It is then easy to see that we must have

$$
\begin{gathered}
w=\rho(\lambda t+x), \\
v=\rho(\lambda x+t) .
\end{gathered}
$$

We require further that $w$ retain its previous significance as Euclidean distance on $P$. Hence for $x, t$ on $P$ we have

$$
\begin{aligned}
t & =\lambda w /\left(\lambda^{2}+1\right)^{1 / 2} \\
& =\lambda w /\left(\rho\left(\lambda^{2}-1\right)\right) .
\end{aligned}
$$

Accordingly $\rho=\left(\lambda^{2}+1\right)^{1 / 2} /\left(\lambda^{2}-1\right)$ and

$$
d \sigma^{2}=\operatorname{sech} \alpha\left(d w^{2}-d v^{2}\right) .
$$

Hereafter $\beta^{2}$ is written for sech $\alpha$ with $\beta>0$.

The $w, v$ coordinates of $x_{0}, t_{0}$ are designated by $w_{0}, v_{0}$. Those of $X_{n}, T_{n}$ and $X_{n}^{\prime}$, $T_{n}^{\prime}$ by $W_{n}, V_{n}$ and $W_{n}^{\prime}, V_{n}^{\prime}$ respectively. When $v_{0}=0$ the distinction between primed and unprimed letters fails and we denote them by $w_{n}$ and $v_{n}$ in the obvious correspondence. We find in a straightforward way that

$$
\begin{aligned}
& W_{n}=(\cosh n \alpha-1) l\left(\lambda^{2}+1\right)^{1 / 2}+w_{0} \cosh n \alpha+v_{0} \sinh n \alpha . \\
& V_{n}=\sinh n \alpha l\left(\lambda^{2}+1\right)^{1 / 2}+v_{0} \cosh n \alpha+w_{0} \sinh n \alpha . \\
& W_{n}^{\prime}=(\cosh n \alpha-1) l\left(\lambda^{2}+1\right)^{1 / 2}+w_{0} \cosh n \alpha-v_{0} \sinh n \alpha . \\
& V_{n}^{\prime}=\sinh n \alpha l\left(\lambda^{2}+1\right)^{1 / 2}+w_{0} \sinh n \alpha-v_{0} \cosh n \alpha .
\end{aligned}
$$

The minus characteristic through $W_{n}, V_{n}$ cuts $v=0$ at $w=w^{n}$ and $x=l$ at $t=$ $t^{n}$. We define $w^{\prime n}, v^{\prime n}$ similarly with respect to the minus characteristic through $W_{n}^{\prime}$, $V_{n}^{\prime}$. Observe that $J^{n-1}$ has $J^{n-1} L$ as its image in $P$ and $J^{n-1} L$ has $J^{n}$ as its image in $P^{\prime}$. It therefore follows from the definition of images that the plus characteristic through $J^{n-1} L$ cuts $P$ at $w=w^{n-1}$ and $x=l$ at $t=t^{n}$. Indeed the hyperbolic distance of $\left(w^{n-1}, 0\right)$ from either $J^{n-1}$ or $J^{n-1} L$ is 0 since characteristics are null geodesics. Analogous remarks hold for the left image points of $K^{n}: W_{n}^{\prime}, V_{n}^{\prime}$.

It is manifest from (2.09) that in the $w, v$ coordinates the characteristics are $w \pm v=$ $C$ and that plus characteristics in the $x, t$ system are again plus in the $w, v$ system. Accordingly ${ }^{6}$

$$
\begin{aligned}
& w^{0}=w_{0}-v_{0}, \\
& w^{n}=W_{n}-V_{n}, \\
& w^{\prime n}=W_{n}^{\prime}-V_{n}^{\prime} .
\end{aligned}
$$

${ }^{6}$ Repeated tacit use is made of (2.12), particularly as regards changing from the sub to superscript 0 and vice versa. 
For $x, t$ on $P$ we have ${ }^{7}$

$$
J_{0}\left[\left(t-T_{n}\right)^{2}-\left(x-X_{n}\right)^{2}\right]^{1 / 2}=J_{0} \beta\left[\left(w-W_{n}\right)^{2}-\left(V_{n}\right)^{2}\right]^{1 / 2}
$$

and a corresponding expression in $W_{n}^{\prime}, V_{n}^{\prime}$ if the image point is $X_{n}^{\prime}, T_{n}^{\prime}$.

3. The associated integral equations. Let $\gamma$ designate the curve $P, P^{\prime}$ (for $t \geq 0$ ) and $P^{\prime \prime}$ where $P^{\prime \prime}$ is the segment of $t=0$ connecting $P$ and $P^{\prime}$. Consider $w_{0}, v_{0}$ or any of the image points $L, R, J^{n}, K^{n}, J^{n} L, K^{n} L$ as an apex for the plus and minus characteristic rays. We need consider only the case where one, at least, of these rays intersects $\gamma$. Then a convex polygon is uniquely determined by these rays and $\boldsymbol{\gamma}$. The boundary of this polygon is denoted by $\Gamma$. For convenience we shall use an obvious mixed notation. Thus if the first argument of $z($,$) is x$, the other is $t$, if the first is $w$ the second is $v$. Suppose $w_{0}, v_{0}$ is the apex. The associated polygon may be five sided. Assume this is indeed the case for the $w_{0}, v_{0}$ chosen. Then by reason of (1.07) and the properties of the Riemann function, (1.04) becomes

$$
\begin{aligned}
2 z\left(w_{0}, v_{0}\right)-z\left(w^{0}, 0\right)-z\left(l, t^{\prime}\right) & \\
& +\int_{0}^{w^{0}}\left[J_{0} \beta\left(w^{0}-w\right) z_{v}(w, 0)-\left.z(w, 0) \frac{\partial}{\partial v} J_{0} \beta\left(w^{0}-w\right)\right|_{P}\right] d w \\
& +\int_{\theta}^{t^{\circ}} z(l, t) \frac{\partial}{\partial x} J_{0}\left[\left(t-t_{0}\right)^{2}-\left(l-x_{0}\right)^{2}\right]^{1 / 2} d t=0 .
\end{aligned}
$$

If an image point is taken as apex then the associated polygon is at most a quadrilateral. Assume this optimum case. Then the vertices of the polygon are at $\left(\omega_{n}, 0\right),\left(l, \tau_{n}\right)$, $(0,0)$ and $(l, 0)$ where the last refers to the $(x, t)$ coordinates. Here $\omega_{n}, \tau_{n}$ is either $w^{n}$, $t^{n} ; w^{\prime n}, t^{\prime n} ; w^{n}, t^{n+1}$ or $w^{\prime n}, t^{\prime n+1}$ depending on whether $J^{n}, K^{n}, J^{n} L$ or $K^{n} L$ is the image point selected as apex.

$$
\begin{aligned}
z\left(\omega_{n}, 0\right)-z\left(l, \tau_{n}\right) & +\int_{0}^{\omega_{n}}\left[J_{0}(\beta D) \frac{\partial}{\partial v} z(w, 0)-\left.z(w, 0) \frac{\partial}{\partial v} J_{0}(\beta D)\right|_{P}\right] d w \\
& +\left.\int_{0}^{\tau_{n}} z(l, t) \frac{\partial}{\partial x} J_{0}(\beta D)\right|_{x=l} d t=0, \quad n \geq 1 .
\end{aligned}
$$

The argument $\beta D$ of $J_{0}(\beta D)$ is $\beta$ times the hyperbolic distance from the apex point to $P$ or $P^{\prime}$ according as the first or second integral is considered in (3.01). We may now waive the requirement that $\omega_{n}, \tau_{n}$ be non negative in (3.0) or (3.01) provided it be understood that a negative integration limit means the integral is 0 and that $z(w, 0)$ and $z(l, t)$ are 0 for $w$ or $t$ negative.

We sum on $n$, the left hand members of (3.01) plus (3.0). This is a finite sum since starting with arbitrary $w_{0}, v_{0}$, eventually $\omega_{n}$ and $\tau_{n}$ are negative. Hence if necessary the order of summing may be modified at will. Manifestly the integrated terms balance out in pairs and one is left with $2 z\left(w_{0}, v_{0}\right)$. The integrals enter the sum in pairs having the same upper limit. Specifically if the upper limit is $w^{n}$ then the pair of integrals arises

${ }^{7}$ For typographical reasons we generally omit a parenthesis and write $J_{0} \beta\left[(w-a)^{2}-b^{2}\right]^{1 / 2}$ in place of $J_{0}\left\{\beta\left[(w-a)^{2}-b^{2}\right]^{1 / 2}\right\}$. 
from the pair of images $J^{n}, J^{n} L$. If the upper limit is $w^{\prime n}$ then the image pair is $K^{n}$, $K^{n} L$, etc. With respect to $t^{n}$ the pairs are $J^{n} L, J^{n+1}$. Now the Riemann functions for the pair $J^{n}, J^{n} L$ for instance have equal values on $P$ and opposite values for the conormal derivatives on $P$. Similar remarks hold for $P^{\prime}$ or for the other image pairs. Accordingly the terms involving the integrands balance out and all integrals along $P^{\prime}$ balance out. Our final result is, then,

$$
\begin{aligned}
-z\left(w_{0}, v_{0}\right)=\int_{0}^{w^{0}} J_{0} \beta[(w & \left.\left.-W_{0}\right)^{2}-\left(V_{0}\right)^{2}\right]\left.^{1 / 2} \frac{\partial z}{\partial v}\right|_{P} d w \\
& +\left.\sum_{n=1} \int_{0}^{w^{n}} J_{0} \beta\left[\left(w-W_{n}\right)^{2}-\left(V_{n}\right)^{2}\right]^{1 / 2} \frac{\partial z}{\partial v}\right|_{P} d w \\
& +\left.\sum_{n=1} \int_{0}^{w^{\prime n}} J_{0} \beta\left[\left(w-W_{n}^{\prime}\right)^{2}-\left(V_{n}^{\prime}\right)^{2}\right]^{1 / 2} \frac{\partial z}{\partial v}\right|_{P} d w .
\end{aligned}
$$

It is convenient for our method of attack on (3.02) that one limit of the integrals occurring be the same for all integrals. Therefore replace $w$ by $w^{0}-w$ in the integral equation above and use (1.07). This leads to our basic formulation

$$
\begin{aligned}
-z\left(w_{0}, v_{0}\right)= & \sum_{n=0} \int_{w^{n}}^{w^{0}} J_{0} \beta\left[\left(w-w^{0}+W_{n}\right)^{2}-\left(V_{n}\right)^{2}\right]^{1 / 2} \frac{\partial z}{\partial v}\left(w^{0}-w, 0\right) d w \\
& +\sum_{n=1} \int_{w^{\prime n}}^{w^{0}} J_{0} \beta\left[\left(w-w^{0}+W_{n}^{\prime}\right)^{2}-\left(V_{n}^{\prime}\right)^{2}\right]^{1 / 2} \frac{\partial z}{\partial v}\left(w^{0}-w, 0\right) d w
\end{aligned}
$$

where

$$
\frac{\partial z}{\partial v}(w, 0)=h z(w, 0)+\psi(w) .
$$

Let the point $w_{0}, v_{0}$ move to the boundary $P$. Then $\left(w_{0}, v_{0}\right) \rightarrow\left(w^{0}, 0\right)$ and in place of (3.03) we have

$$
\left.-z\left(w^{0}, 0\right)=1+2 \sum_{n=1} \int_{w^{n}}^{w^{0}} J_{0} \beta\left[\left(w-w^{0}+w_{n}\right)^{2}-\left(v_{n}\right)^{2}\right)\right]^{1 / 2}(h z(w, 0)+\psi(w)] d w .
$$

4. Problem 2. Our second problem may now be set up with ease on the basis of the formulation for the first problem. Here $P^{\prime}$ is the line $t+\lambda x=\lambda l$.

A simple observation enables us to determine the image points $(L R)^{n}, K^{n}$ with a minimum of effort. Consider for a moment (2.03) where now $x^{\prime}, t^{\prime}$ is the object and $x_{0}, t_{0}$ the image. Write

$$
\mathbf{N}=\left(\begin{array}{ll}
-\cosh \alpha & \sinh \alpha \\
-\sinh \alpha & \cosh \alpha
\end{array}\right)
$$

Then

$$
\left(x_{0} t_{0}\right)=\left(x^{\prime} t^{\prime}\right) \mathbf{N}^{-1}
$$


If we seek the reflection in $P^{\prime}$ we need merely replace $x_{0}, x^{\prime}$ by $x_{0}-l$ and $x^{\prime}-l$ respectively. That is to say

$$
\left(x_{0}-l t_{0}\right)=\left(x^{\prime}-l t^{\prime}\right) \mathbf{N}^{-1} .
$$

If $x^{\prime}, t^{\prime}$ are the coordinates of $L$ and $X_{1}, T_{1}$ are the coordinates of $L R$ then we have

$$
\left(X_{1}-l T_{1}\right)=\left(x_{0} t_{0}\right) \mathbf{N N}^{-1}-(l 0) \mathbf{N}^{-1},
$$

or

$$
X_{1}=l(1+\cosh \alpha)+x_{0}, \quad T_{1}=-l \sinh \alpha+t_{0} .
$$

Hence $X_{n}, T_{n}$ the coordinates of $(L R)^{n}$ are, evidently,

$$
\begin{aligned}
& X_{n}=n l(1+\cosh \alpha)+x_{0}, \\
& T_{n}=-n l \sinh \alpha+t_{0} .
\end{aligned}
$$

It is easy to see that the coordinates $X_{n}^{\prime}, T_{n}^{\prime}$ of $R(L R)^{n}$ are, in view of (2.03),

$$
\begin{aligned}
X_{n}^{\prime} & =(n+1) l(1+\cosh \alpha)-\left(x_{0} \cosh \alpha+t_{0} \sinh \alpha\right), \\
T_{n}^{\prime} & =-(n+1) l \sinh \alpha+x_{0} \sinh \alpha+t_{0} \cosh \alpha .
\end{aligned}
$$

We now pass to the $w, v$ coordinates using the same letters $W_{n}, V_{n}, \cdots$ as before for the corresponding coordinates of $J^{n}, \cdots$. Then with $w_{0}, v_{0}$ as the $w, v$ coordinates for $x_{0}, t_{0}$ and our earlier definition of $\rho$ we have

$$
\begin{aligned}
& W_{n}=W_{0}=W^{\prime}=w_{0} . \\
& V_{n}=2 \lambda n l_{\rho}+v_{0} . \\
& V_{n}^{\prime}=2 \lambda(n+1) l_{\rho}-v_{0} .
\end{aligned}
$$

We write

$$
\begin{aligned}
w^{0} & =w_{0}-v_{0} \\
{w^{\prime}}^{0} & =w_{0}+v_{0}-2 l \rho \lambda .
\end{aligned}
$$

The intersections of the plus characteristics through $(L R)^{n}$ and $R(L R)^{n}$ with $P$ are denoted by $\left(w^{n}, 0\right)$ and $\left(w^{\prime n}, 0\right)$ respectively where

$$
\begin{aligned}
w^{n} & =W_{n}-V_{n}=w^{0}-2 \lambda n l \rho \\
w^{\prime n} & =w^{\prime 0}-2 \lambda n l_{\rho}
\end{aligned}
$$

We take over (3.03), (3.05) with the understanding that the symbols now be interpreted according to (4.03). Thus (3.03) is now

$$
\begin{gathered}
-z\left(w_{0}, v_{0}\right)=\sum_{0}^{\infty} \int_{2 \lambda_{n} \ell \rho}^{w^{\circ}} J_{0} \beta\left[\left(w+v_{0}\right)^{2}-\left(2 \lambda n l_{\rho}+v_{0}\right)^{2}\right]^{1 / 2} z_{v}(w, 0) d w \\
+\sum_{1}^{\infty} \int_{2 \lambda(n+1) \iota \rho-2 v_{0}}^{w^{\circ}} J_{0} \beta\left[\left(w+v_{0}\right)^{2}-\left(2 \lambda(n+1) l_{\rho}-v_{0}\right)^{2}\right]^{1 / 2} z_{v}(w, 0) d w, \\
z_{v}(w, 0)=h z(w, 0)+\psi(w)
\end{gathered}
$$


We refer to (4.06) with $v_{0}=0$ and $z_{\nu}(w, 0)$ given by (4.07) as (4.08).

5. Transforms. We bring together here some explicit evaluations of the inverse Laplace transforms arising in our problems. Let $q(w)$ have the Laplace transform $Q(p)$. Consider

$$
\frac{1}{2 \pi i} \int_{d-i \infty}^{d+i \infty} e^{w p} Q(p) d p
$$

This integral is interpreted as a Cauchy principal value. For $d$ sufficiently large and under certain well known conditions the integral exists and yields $[q(w+0)+$ $q(w-0)] / 2$. We indicate the reciprocal correspondence between $q(w)$ and $Q(p)$ by $Q(p) \sim q(w)$ and refer to $q(w)$ as the inverse transform of $Q(p)$ (with respect to the kernel exp (wp)).

A standard result for $B \geq 0$ is ${ }^{8}$

$$
\begin{aligned}
\exp (-B q) / q & \sim J_{0} \beta\left(w^{2}-B^{2}\right)^{1 / 2}, \quad w>B, \\
& \sim 0, \quad w<B
\end{aligned}
$$

We use the symbol $q$ for $\left(p^{2}+\beta^{2}\right)^{1 / 2}$ throughout this paper. It is therefore trivial that for $B \geq v_{0}$ and only then,

$$
\begin{aligned}
\exp \left(v_{0} p-B q\right) / q & \left.\sim J_{0} \beta\left(w+v_{0}\right)^{2}-B^{2}\right)^{1 / 2}, & & w>B-v_{0} \\
& \sim 0 & & w<B-v_{0}
\end{aligned}
$$

where $B$ is either $V_{n}$ or $V_{n}^{\prime}$.

The typical inverse transform of concern to us is that of

$$
\left[\exp \left(v_{0} p-B q\right)\right] /(q+h)^{n} .
$$

with respect to the kernel exp $w p$. The presence of $q$ in the function suggests the natural substitution

$$
p=i \beta \cos (\sigma+i \tau), \quad 0 \leq \sigma \leq \pi .
$$

(With this substitution, eventually an integral over a finite contour is obtained so that our manipulations are evidently valid. This would not be quite so obvious if we used (5.0) directly to obtain (5.09)). The path of integration in (5.0) then becomes the contour $C$ defined by

$$
\beta \sin \sigma \sinh \tau=d
$$

This is a loop lying between $\sigma=0$ and $\sigma=\pi$ with minimum at height $\operatorname{arc} \sinh d / \beta$ and asymptotic to $\sigma=0$ and $\sigma=\pi$. Then (5.0) becomes, with $\phi=\sigma+i \tau$ and $u=$ $w+v_{0}$,

$$
\frac{1}{2 \pi} \int_{0}^{\pi} \exp [\beta(i u \cos \phi-B \sin \phi)] \sin \phi /(\beta \sin \phi+h)^{n} d \phi .
$$

For $\tau \uparrow \infty$ the expression $\sin \sigma(u \sinh \tau-B \cosh \tau)$ goes to $-\infty$ when $0<\sigma<\pi$ and

${ }^{8}$ R. V. Churchill, Modern operational mathematics in engineering, McGraw-Hill, 1944. 
$B>u$ or when $-\pi<\sigma<0$ and $u>B$. It is then easy to see that the integral in (5.04) vanishes for $B>u$.

For the case $u>B$ we note that the integral over $C_{r}$, the reflection of $C$ in $\sigma=0$, is 0 and hence for this case $C$ may be replaced by $C^{\prime}=C+C_{r}$. Evidently the singularities are at the points

$$
\begin{aligned}
\beta \sin \sigma \cosh \tau & =-h, \\
\cos \sigma \sinh \tau & =0 .
\end{aligned}
$$

For $d$ sufficiently large and some non negative $\delta, \delta<d$, the imaginary parts of the singularities are inferior to $\delta$. Consider the closed contour determined by the verticals at $\sigma=-\pi$ and $\sigma=\pi$, the line $\tau=\delta$ between $\sigma=-\pi$ and $\sigma=\pi$ and $C^{\prime}$. Evidently the contributions for the two verticals balance each other. Hence the integral in (5.04) is

$\frac{1}{2 \pi} \int_{-\pi}^{\pi} \exp [\beta\{i u \cos (\sigma+i \delta)-B \sin (\sigma+i \delta)\}]$

$$
\sin (\sigma+i \delta) /(\beta \sin (\sigma+i \delta)+h)^{n} d \sigma
$$

Let

$$
\begin{gathered}
Q(u, B)=\frac{1}{2 \pi} \int_{-\pi}^{\pi}[\exp [\beta\{i u \cos (\sigma+i \delta)-B \sin (\sigma+i \delta)\}] \\
\left./(\beta \sin (\sigma+i \delta)+h)^{n}\right] d \sigma
\end{gathered}
$$

We write $Q^{i}(B, B)$ for $\partial^{i} Q(u, B) / \partial u^{i}$ at $u=B$, and $z$ for $\exp (i \sigma-\delta)$. Then

$$
-i \pi 2^{1-n} Q(B, B)=\int_{D}\left[\exp (i \beta B z) z^{n-1} /\left\{\beta\left(z^{2}-1\right)+2 h i z\right\}^{n}\right] d z .
$$

Here the contour $D$ is the circle of radius $\exp (-\delta)$. Moreover

$$
-i \pi 2^{1+j-1}(i \beta)^{-i} Q^{i}(B, B)=\int_{D}\left[\exp (i \beta B z)\left(z^{2}+1\right)^{i} z^{n-i-1} /\left\{\beta\left(z^{2}-1\right)+2 h i z\right\}^{n}\right] d z .
$$

Plainly $Q^{i}(B, B)=0$ for $j \leq n-1$ since the integrand is then regular and analytic. Evidently too, $\partial^{i} Q(u, B) / \partial B^{i}=0$ for $u=B, j \leq n-1$ by the same type of argument. We next observe that partial differentiation of $Q(u, B)$ with respect to $B$ introduces a factor of $-\beta \sin (\sigma+i \delta)$ in the integrand. Hence

$$
\begin{gathered}
\left(-\frac{\partial}{\partial B}+h\right)^{n} Q(u, B)=\frac{1}{2 \pi} \int_{-\pi}^{\pi} \exp [\beta(i u \cos (\sigma+i \delta)-B \sin (\sigma+i \delta)] d \sigma \\
=J_{0} \beta\left(u^{2}-B^{2}\right)^{1 / 2} . \\
Q^{i}(B, B)=0=\partial^{i} Q(B, B) / \partial u^{i}, \quad j \leq n-1 .
\end{gathered}
$$

It is not difficult to solve (5.08). Thus let $u$ be interpreted for the moment as a fixed parameter. In order to obtain a solution satisfying the boundary conditions, (5.09), the Laplace transform technique seems natural. However, the facts that $B<u$ and 
the differential operator involves $-\partial / \partial B$ require that the Laplace transform be over the variable range $-\infty$ to $u$ and not over $u$ to $\infty$. This implies obvious changes in the expressions for the convolutions. (An alternative method which is essentially equivalent is to replace $u, B$ by $-\mu,-b$. The usual convolution theory may then be applied): In any case it will be perceived that the result is

$$
Q(u, B)=\frac{1}{\Gamma(n)} \int_{B}^{u}(s-B)^{n-1} e^{-h(s-B)} J_{0} \beta\left(u^{2}-s^{2}\right)^{1 / 2} d s .
$$

The integral in (5.04) is evidently $-\partial Q(u, B) / \partial B$ and is therefore

$$
\begin{array}{cc}
\frac{1}{\Gamma(n)} \int_{B}^{u}(s-B)^{n-2}[n-1-h(s-B)] & \\
\exp [-h(s-B)] J_{0} \beta\left(u^{2}-s^{2}\right)^{1 / 2} d s, & n>1 \\
J_{0} \beta\left(u^{2}-B^{2}\right)^{1 / 2}-h / \Gamma(n) \int_{B}^{u} \exp \left[-h(s-B] J_{0} \beta\left(u^{2}-s^{2}\right)^{1 / 2} d s,\right. & n=1
\end{array}
$$

Consider now

$$
M(p)=-h /(q+h)
$$

The inverse transformation of $M(p)$ is obtained as follows. Consider

$$
M(p, \epsilon)=M(p) \exp (-\epsilon q), \quad \epsilon>0 .
$$

The Plancherel theorem, for instance, asserts that in the $L_{2}$ sense the limit of the inverse transform of $M(p, \epsilon)$ is the inverse transform of $M(p)$. Hence we should expect from (5.12) that

$$
M(p) \sim-h J_{0} \beta w+h^{2} \int_{0}^{w} e^{-h s} J_{0} \beta\left(w^{2}-s^{2}\right)^{1 / 2} d s
$$

We need bother with no abstract arguments since it is easy to verify that the Laplace transform of the right hand side of (5.13) is actually $M(p)$. Indeed this follows from

$$
\begin{aligned}
\int_{0}^{\infty} \int_{0}^{w} e^{-h s} J_{0} \beta\left(w^{2}-s^{2}\right) d s e^{-p w} d w & =\int_{0}^{\infty} \int_{s}^{\infty} e^{-p w} J_{0} \beta\left(w^{2}-s^{2}\right)^{1 / 2} d w e^{-h s} d s \\
& =\int_{0}^{\infty}\{\exp [-(h s+q s)] / q\} d s=[q(q+h)]^{-1} .
\end{aligned}
$$

6. Solution of problem 2. The integrals in (4.06) for fixed $v_{0}$ or in (4.08) are evidently tonvolutions and so the Laplace transform theory may be applied. The integrals in (3.03), (3.05), (4.06) and (4.08) are understood to vanish when the lower limit is negacive. Actually no special care in our manipulations is required to ensure this for, on passing to the Laplace transforms, (5.01) guarantees the irrelevancy of integrals for which $V_{n}-v_{0}$ or $V_{n}^{\prime}-v_{0}$ dominates $w^{0}$.

We proceed formally at first and reserve the rigorous establishment of the validity of our analysis to a later place. Write 


$$
\begin{aligned}
& F(p) \sim z(w, 0) \\
& H\left(p, v_{0}\right) \sim z\left(w, v_{0}\right) \\
& D(p) \sim \psi(w) \\
& Q_{M}(p)=\left[1+2 \sum_{1}^{M} \exp (-2 l \lambda \rho n q)\right] q^{-1} \\
& P_{N}(p)=q^{-1} \exp v_{0} p \sum_{0}^{N}\left\{\exp \left[-\left(v_{0}+2 \lambda l \rho n\right) q\right]-\exp \left[v_{0}-2 \lambda l \rho(n+1) q\right]\right\}
\end{aligned}
$$

It will then be clear that (4.08) and (4.06) imply

$$
\begin{aligned}
F(p) & =-[h F(p)+D(p)] Q_{M}(p), \\
H\left(p, v_{0}\right) & =-[h F(p)+D(p)] P_{N}(p) .
\end{aligned}
$$

Strictly speaking $F(p)$ and $H\left(p, v_{0}\right)$ depend on $M$ and $N$ respectively. However, in view of a previous italicized remark, for prescribed $w_{0}$ and all $M$ and $N$ sufficiently large, the inverse transforms for $w=w^{0}$ are the same. To indicate this special choice of $w$ we write $\sim w_{0}$ in place of $\sim$. The admissible integers $M$ are evidently those at least as great as $w^{0} /(2 l \lambda \rho)$ and $N$ may be chosen equal to $M$. We next remark that since $\psi(w) \varepsilon C^{\prime}$ we may if necessary modify it in the range $2 w_{0} \leq w<\infty$ so that the modified $\psi(w)$ belongs to $C^{\prime}$ and its derivative is Lebesgue summable on $0 \leq w<\infty$. The transform $D(p)$ is tacitly understood in the sequel to refer to the modified function. Of course, the modifications have no influence on the $\sim w_{0}$ relations.

Our key relation is the consequence of (6.01) namely

$$
\begin{aligned}
z\left(w_{0},\right. & \left.v_{0}\right) \sim H\left(p, v_{0}\right) \\
& =-D(p) P_{N}(p)\left[1+h Q_{N}(p)\right]^{-1} \\
& =\frac{-D(p) \exp \left(v_{0} p\right)\left[\exp \left(-v_{0} q\right)+\exp \left(v_{0}-2 l \lambda \rho\right) q\right] \sum_{0}^{N} \exp (-2 \lambda l \rho n q)}{(q+h)+2 h \sum_{1}^{N} \exp (-2 l \lambda \rho n q)} .
\end{aligned}
$$

Since no error is committed by allowing $N$ to increase without limit, we have

$$
H\left(p, v_{0}\right)=\frac{-D(p) \exp \left(v_{0} p\right)\left[\exp \left(-v_{0} q\right)+\exp \left\{\left(v_{0}-2 l_{\rho \lambda}\right) q\right\}\right]}{(q+h)+(h-q) \exp \left(-2 l_{\rho \lambda q)}\right.}
$$

However, in the calculation of the inverse transform we use the expression essentially equivalent to (6.02).

(Actually (6.03) may be derived by the usual transform method though slight generalization such as that involved in the passage to our main problem eliminates this as a general method. Thus we write (1.0) in $v, w$ coordinates. Consider the sector determined by $v=\rho l, v-w=\rho l(\lambda-1)$ with $z_{v}=0$ on the first and $z=0$ on the second. The conditions imply, as is well known, that $z \equiv 0$ in the sector. The Cauchy problem with vanishing data on $t=0$ implies a vanishing solution in the triangle bounded by $t=0, v+w=0, v-w=\rho l(\lambda-1)$. In particular $z=0$ on $v-w=$ 
$\rho l(\lambda-1)$. Hence we know that $z=0$ for the triangle bounded by $t=0, v+w=0$ and $v=\rho l$. The solution of our original problem is, therefore, unaffected if we replace the vanishing Cauchy data on $t=0$ by 0 data on $w=0$. We have now the problem of satisfying

$$
\begin{aligned}
& u_{v v}-\left(p^{2}+\beta^{2}\right) u=0 \\
& u_{v}=h u+\psi \text { on } v=0 \\
& u=u_{v}=u_{w}=0 \text { on } w=0 \\
& u_{v}=0 \text { on } v=\rho l .
\end{aligned}
$$

where $u$ is the Laplace transform with respect to $w$ of $z(w, v)$. Evidently $u$ is precisely our $H\left(p, v_{0}\right)$ and one easily verifies that the system above leads to (6.03). Of course a similar remark holds for the simpler situation treated by Chandrasekhar).

It is convenient to write $S(p)$ for the reciprocal of the denominator in (6.03) and $T(p)$ for the numerator divided by $D(p)$. Let ${ }^{9}$

$$
\begin{aligned}
S_{N}(p) & =(q .+h)^{-1} \sum_{0}^{N}\left(\frac{q-h}{q+h}\right)^{i} e^{-2 l \rho \lambda j q} \\
& =\sum_{0}^{N}\left(\sum_{0}^{i}{ }_{i} C_{k}(-2 h)^{k}(q+h)^{-k-1}\right) e^{-2 l \rho \lambda i \rho} .
\end{aligned}
$$

Write

$$
\begin{aligned}
H_{N}(p) & =H\left(p, v_{0}\right) S_{N}(p) / S(p) \\
K(p) & =H\left(p, v_{0}\right) / D(p) \\
K_{N}(p) & =K(p)\left(1-S_{N}(p) / S(p)\right) \\
& =\sum_{0}^{N+1}{ }_{N+1} C_{k}(-2 h)^{k}(q+h)^{-k} S(p) T(p) e^{-2 l \rho(N+1) \lambda} \\
& =S(p) T(p) e^{-2 l \rho(N+1) \lambda a}+k_{N}(p)
\end{aligned}
$$

We show now that

$$
K_{N}(p) \sim w_{0} 0
$$

for admissible $N$ values.

The following relations are easy to establish for $R(p)$ large enough

$$
\begin{aligned}
|q+| h|| & \geq|p|, \\
|q-| h|| & \leq|p|, \\
R(q) & \geq R(p) .
\end{aligned}
$$

${ }^{9}$ The symbol ${ }_{N} C_{i}$ is the familiar binomial coefficient. 
In view of (6.04) it is plain that for $h>0$

$$
|S(p)| \leq\left[|p|\left(1-\exp -2 l_{\rho} \lambda R(p)\right)\right]^{-1} \leq 2|p|^{-1},
$$

for $R(p)$ sufficiently large. That is to say $|S(p)|=0\left(|p|^{-1}\right)$ as $|p| \rightarrow \infty$ at least in some half plane to the right of the imaginary axis. The order relation for $S(p)$ together with (6.04) guarantees there are no polar singularities far enough to the right. We need one more fact, namely that

$$
|\exp (a p-b q)| \leq \exp [(a-b) R(p)]
$$

We apply this to

$$
T(p) e^{v_{0} p-2 l \lambda \rho(N+1) a}
$$

and note that in view of the definition of $N$, (6.05) applies with $a<b$.

Observe

$$
S(p)=\left[q\left(1-e^{-2 l \lambda \rho q}\right)\right]^{-1}-h S(p)\left(1+e^{-2 l \lambda \rho q}\right)\left[q\left(1-e^{-2 l \rho \lambda q}\right)\right]^{-1}
$$

The second term above is $0|p|^{-2}$ for $R(p) \geq d$. The first term on the right hand side has an inverse transform interpreted as a Cauchy principal value which is in fact a finite sum of Bessel's functions for any positive $w$ and reciprocally the Laplace transform of this inverse transform is precisely the first term. It is then trivial that $S(p) T(p)$ $\exp -2 l \rho(N+1) q \lambda$ admits an inverse transform and, since $k_{N}(p)$ is regular and is $0\left(|p|^{-2}\right)$ for $R(p) \geq d$, the same is true of $k_{N}(p)$. In short, $K_{N}(p)$ admits an inverse transform. ${ }^{10}$ That $K_{N}(p) \sim w_{0} 0$ is now an easy consequence of the fact that the real part of $w_{0} p-2 l_{\rho}(N+1) \lambda q$ is negative for $R(p) \geq d$.

On writing $(q+h)^{-1}$ as

$$
q^{-1}-h[(q+h) q]^{-1}
$$

it is easy to show, by reasoning similar to that above, that the inverse transform of $K(p) S_{N}(p) / S(p)$ exists. The trivial identity

$$
K(p)=K_{N}(p)+\left[K(p)-K_{N}(p)\right]=K_{N}(p)+H_{N}(p) / D(p)
$$

shows that the inverse transform of $K(p)$ exists and for $w \leq w_{0}$ is identical with that of $H_{N}(p) / D(p)$.

In view of (5.11) and (5.12) we may write, with $w$ replacing $w_{0}$,

$$
\begin{aligned}
& g\left(w, v_{0}\right) \sim H\left(p, v_{0}\right) / D(p) \\
&=-\left[\sum_{0}^{N} \sum_{i}^{k}{ }_{1} C_{k}(-2 h)^{i}(\Gamma(k+1))^{-1}\right. \\
&\left\{\int_{V_{i}}^{w}\left(s-V_{j}\right)^{k-1}\left[h\left(s-V_{i}\right)-k\right] e^{-h\left(s-V_{i}\right)} J_{0} \beta\left(w^{2}-s^{2}\right)^{1 / 2} d s\right. \\
&+\left.\int_{V^{\prime} j}^{w}\left(s-V_{i}^{\prime}\right)^{k-1}\left[h\left(s-V_{j}^{\prime}\right)-k\right] e^{-h\left(s-V^{\prime}{ }^{\prime}\right)} J_{0} \beta\left(w^{2}-s^{2}\right)^{1 / 2} d s\right\}
\end{aligned}
$$

${ }^{10} \mathrm{G}$. Doetsch, Theorie und Anwendung der Laplace Transformation, Dover Publications, 1943. Cf. in particular p. 128. 


$$
\begin{aligned}
& +\sum_{0}^{N}\left\{J_{0} \beta\left(w^{2}-V_{j}^{2}\right)^{1 / 2}+J_{0} \beta\left(w^{2}-V_{i}^{\prime 2}\right)^{1 / 2}\right. \\
& \left.\left.+h \int_{V_{i}}^{w} e^{-h\left(s-V_{j}\right)} J_{0} \beta\left(w^{2}-s^{2}\right)^{1 / 2} d s+\int_{V^{\prime}{ }_{i}}^{w} e^{-h\left(s-V^{\prime}{ }^{\prime}\right)} J_{0} \beta\left(w^{2}-s^{2}\right)^{1 / 2} d s\right\}\right] .
\end{aligned}
$$

Again we observe that the terms with $V_{i}, V_{i}^{\prime}>w$ are to be replaced by 0 . Then

$$
z\left(w_{0}, v_{0}\right)=\int_{0}^{w_{0}} \psi\left(w_{0}-w\right) g\left(w, v_{0}\right) d w .
$$

Equation (6.09) gives the formal solution of Problem 2. The verification of this fact will be given in Sec. 8 .

7. Problem 1. The integrals occurring in (3.03) or (3.05) are not convolutions since the variable $w^{0}$ occurs in $V_{n}, V_{n}^{\prime}$. Nevertheless they may be evaluated in terms of convolutions even though paradoxically the equations cannot be directly solved this way. Since we make use of these observations it is worthwhile giving more detail.

The typical integral in (3.03) or (3.05) is of the form

$$
\int_{w^{0}-w^{n}}^{w_{n}} J_{0} \beta\left[\left(w+W_{n}-w^{0}\right)^{2}-\left(V_{n}\right)^{2}\right]^{1 / 2} f\left(w^{0}-w\right) d w=I\left(w^{0}\right) .
$$

We propose to replace $w^{0}$ by a new variable $t$ in the limits and in the argument of $f(\quad)$ but not in $W_{n}-w^{0}$ or $V_{n}$ so that we obtain an integral of convolution type depending on a parameter $w^{0}$. Thus, consider

$$
I\left(w^{0}, t\right)=\int_{t-w^{n}}^{t} E\left(s, w^{0}\right) f(t-s) d s
$$

where

$$
\begin{array}{rlrl}
E\left(s, w^{0}\right) & =J_{0} \beta\left[\left(s+W_{n}-w^{0}\right)^{2}-\left(V_{n}\right)^{2}\right]^{1 / 2}, & & s>w^{0}-w^{n} \\
& =0 \quad, \quad & s<w^{0}-w^{n}
\end{array}
$$

Evidently for $t<w^{0}$ we can express $I\left(w^{0}, t\right)$ as a convolution

$$
\left(w^{0}, t\right)=\int_{0}^{t} E\left(s, w^{0}\right) f(t-s) d s
$$

Moreover

$$
L_{t \rightarrow w^{\circ}} I\left(w^{0}, t\right)=I\left(w^{0}, w^{0}\right)=I\left(w^{0}\right) .
$$

Indeed the convolution is evaluated as the inverse transform with kernel exp $\left(w^{0} p\right)$ of

$$
\begin{gathered}
e^{\left(w_{n}-w^{0}\right) p-V_{n a}} F(p) q^{-1}, \\
F(p) \sim w^{0} f\left(w^{0}\right)
\end{gathered}
$$

More accurately the inverse transform is taken with respect to exp $(t p)$ and then $t$ is given the special value $w^{0}$.

The reason that we cannot argue that (3.03) or (3.05) can be solved by the Laplace transform method in spite of the above is this. Suppose we were to attempt to exploit 
the idea involved in passing from (7.0) to (7.01). For simplicity consider (3.05). We should replace this by the integral equation in which the terms are of type (7.01). We refer to this equation as (3.05)'. This new integral equation determines a solution which depends on $t$ and on the parameter $w^{0}$ so that we should write $Z\left(t, 0, w^{0}\right)$. Let

$$
F\left(p, w^{0}\right) \sim Z\left(t, 0, w^{0}\right) .
$$

Formally at least we could solve for $F\left(p, w^{0}\right)$ from $(3.05)^{\prime}$. We should hope that $Z\left(w^{0}, 0, w^{0}\right)$ is the $z\left(w^{0}, 0\right)$ satisfying $(3.05)$, but this is in general untrue. Actually it is obvious $Z\left(w^{0}, 0, w^{0}\right)$ would satisfy the integral equation of type (3.05) with $z\left(w^{0}-s, 0\right)$ replaced by $Z\left(w^{0}-s, 0, w^{0}\right)$ and not by $Z\left(w^{0}-s, 0, w^{0}-s\right)$. Perhaps the following :simple illustration will be helpful. Consider

$$
z(w)=1+\int_{0^{*}}^{w} w z(w-s) d s
$$

Let $Y(p) \sim z(w)$. It is true that

$$
\left.\int_{0}^{t} w z(t-s) d t\right|_{t=w} \sim w Y(p) .
$$

On the other hand the attempted solution would be

$$
\left.z(w) \sim(p-w)^{-1} \sim e^{-t w}\right|_{t=w}=e^{-w^{2}}
$$

while it is plain that exp $-w^{2}$ certainly does not satisfy (7.02). In fact, the operational interpretation of $w Y(p)$ is really $-d / d p Y(p)$.

Let $y^{0}$ denote the value of $w^{0}$ when the corresponding $w^{1}$ is 0 . Let $y^{n}$ be related to $y^{n-1}$ in the same way as $w^{0}$ to $w^{1}$. That is to say $y^{n}$ is the largest $w^{0}$ value for which only the images $\left\{J^{i} \mid i=1, \cdots n\right\}$ need be taken into account. Then from (2.12) it is patent that

$$
y^{n}=\left(e^{n \alpha}-1\right)\left(\lambda^{2}+1\right)^{1 / 2} l .
$$

Accordingly we have from (3.05) that

$$
\begin{aligned}
& z\left(w^{0}, 0\right)=-\int_{0}^{w^{0}} J_{0} \beta\left(w^{0}-w\right)[h z(w, 0)+\psi(w)] d w \quad w^{0} \leq y^{0} \\
& \ldots \ldots \ldots \ldots \ldots \ldots \ldots \ldots \ldots \ldots \ldots \ldots \ldots \ldots \ldots \ldots \ldots \ldots \ldots \ldots \ldots \ldots \ldots \ldots \ldots \ldots \ldots \ldots \ldots \\
&=-\left[\int_{0}^{y^{n-1}}+\int_{y^{n-1}}^{w^{0}} J_{0} \beta\left(w^{0}-w\right)[h z(w, 0)+\psi(w)] d w\right. \\
&+2 \sum_{i=1}^{n} \int_{0}^{w_{i}} J_{0} \beta\left[\left(w-w_{i}\right)^{2}-\left(v_{i}\right)^{2}\right]^{1 / 2}[h z(w, 0)+\psi(w)] d w, \\
& y^{n-1} \leq w^{0} \leq y^{n} .
\end{aligned}
$$

We refer to the value of $z\left(w^{0}, 0\right)$ in the range $y^{n-1} \leq w^{0} \leq y^{n}$ as $z_{n}\left(w^{0}\right)$. We solve the first equation for $z_{0}\left(w^{0}\right)$. In the equation over the range $y^{0} \leq w^{0} \leq y^{1}$ this solution $z_{0}\left(w^{0}\right)$ is used wherever the integration range involved only $w$ values up to $y^{0}$. We proceed step by step. Evidently the only integral involving $z(w, 0)$ for $w$ values surpassing $y^{0}$ is 


$$
-\int_{y^{\circ}}^{w^{\circ}} J_{0} \beta\left(w^{0}-w\right) h z(w, 0) d w
$$

In the general case, for $z_{n}(w)$ we have an integral equation of the form

$$
z_{n}\left(w^{0}, 0\right)=-\int_{y^{x-1}}^{w^{0}} J_{0} \beta\left(w^{0}-s\right) h z_{n}(s, 0) d s+f_{n}(w)
$$

where $f_{n}(w)$ is known since it involves integrals either of $\psi(w)$ or of $z(w)$ over rangescontained in $0 \leq w \leq y^{n-1}$ and these are known from the preceding solutions. In short, then, we have merely to establish the form of the solution of (7.05) and then to apply it at each stage (for the new values of $y^{n-1}$ and $f_{n}(w)$ to obtain the solution of (7.04). The solution of (7.05) can be written

$$
z(w, 0)=f_{n}(w)+\int_{y^{n-1}}^{w} k(w-s, h) f_{n}(s) d s .
$$

We understand below that the inverse transform is to be taken with respect to the kernel $\exp ((w-s) p)$. We start with

$$
q^{-1} \sim J_{0} \beta(w-s), \quad w-s>0
$$

Write $M(p)$ for the transform of $k(w-s, h)$. Then

$$
\begin{aligned}
M(p)=\sum_{1}^{\infty}(-h / q)^{j} & =-h /(q+h) \\
& \sim k(w-s, h) .
\end{aligned}
$$

Hence in view of (5.13) the reciprocal kernel, $k(w-s, h)$, is known. Since $f_{0}(w)$ is known, Cf. (8.01), $z_{0}(w)$ is given by (7.06). Therefore $f_{1}(w)$ is determined, Cf. (8.01), and so (7.06) yields $z_{1}(w)$, etc. Accordingly we may write down the explicit solution for $z(w, 0)$. On substituting this solution in (3.03) we have the formal solution of our problem, namely the value of $z\left(w_{0}, v_{0}\right)$.

8. Validation of the solutions. In this section we verify that the solutions of the two problems of this paper are actually given by Secs. 6 and 7. We consider Problem 1 first and Sec. 7. We observe first that on replacing $z(w, 0)$ by an arbitrary function, restricted merely by suitable continuity conditions, the right hand side of (3.03) satisfies (1.0). It is a straightforward exercise to show that $f_{N}(w) \varepsilon C^{\prime}$ implies by (7.06) and (5.13) that $z(w, 0) \varepsilon C^{\prime}$ for $y^{n-1} \leq w \leq y^{n}$. However $\psi(w) \varepsilon C^{\prime}$ guarantees

$$
f_{0}(w)=-\int_{0}^{w} J_{0} \beta(w-s) \psi(s) d s
$$

belongs to $C^{\prime}$ and hence by successive stages we arrive at $f_{n}(w) \varepsilon C^{\prime}$. We next show that $z(w) \varepsilon C^{\prime}$ at the points $y^{n}$. We observe that

$$
\begin{aligned}
f_{n+1}(w)=f_{n}(w)- & h \int_{y^{n-1}}^{y^{n}} J_{0} \beta(w-s) z_{n}(s) d s \\
& -\int_{0}^{w^{n+1}} J_{0} \beta\left[\left(w-w_{n+1}\right)^{2}-\left(v_{n+1}\right)^{2}\right]^{1 / 2}[h z(w, 0)+\psi(w)] d w .
\end{aligned}
$$


That $z_{n+1}\left(y^{n}\right)=z_{n}\left(y^{n}\right)$ is an immediate consequence of (5.13), (8.01) and the fact that $w^{n+1}=0$ for $y^{n}=w$. From (7.05) one derives

$$
\begin{aligned}
z_{n+1}^{\prime}\left(y^{n}\right) & =-h z_{n+1}\left(y^{n}\right)+f_{n+1}^{\prime}\left(y^{n}\right) \\
& =-h z_{n}\left(y^{n}\right)+f_{n+1}^{\prime}\left(y^{n}\right)
\end{aligned}
$$

where primes here denote differentiation. Since $z(0,0)$ and $\psi(0)$ are zero it is easy to see that the $w$ derivative of the last integral on the right hand side of (8.01) goes to 0 as $w$ goes to $y^{n}$. Accordingly (8.01) implies

$$
f_{n+1}^{\prime}\left(y^{n}\right)=f_{n}^{\prime}\left(y^{n}\right)-h \int_{y^{n-1}}^{y^{n}} J_{0}^{\prime} \beta\left(w^{n}-s\right) z_{n}(s) d s .
$$

Again (7.05) leads to

$$
z_{n}^{\prime}\left(y^{n}\right)=-h z_{n}\left(y^{n}\right)-h \int_{y^{n-1}}^{y^{n}} J_{0}^{\prime} \beta\left(y^{n}-s\right) z_{n}(s) d s+f_{n}^{\prime}\left(y^{n}\right)
$$

and hence by (8.02) and (8.03) we can assert $z_{n}^{\prime}\left(y^{n}\right)=z_{n+1}^{\prime}\left(y^{n}\right)$.

Since then $z(w, 0) \varepsilon C^{\prime}$ and $\psi(w) \varepsilon C^{\prime}$ it follows at once from (3.04) that the expression on the right hand side of (3.02) or (3.03) is of class $C^{2}$ away from the boundary and of class $C^{\prime}$ if the boundary is included. Indeed consider a typical integral

$$
\int_{0}^{w^{n}} J_{0} \beta\left(\left(w-W_{n}\right)^{2}-\left(V_{n}\right)^{2}\right)^{1 / 2} \gamma(w) d w=Y\left(w_{0}, v_{0}\right)
$$

and let primes denote differentiation with respect to $w_{0}$ or $v_{0}$ at will. Then since $\left(w^{n}\right)^{\prime \prime}=0$ we have

$$
\begin{aligned}
& Y^{\prime \prime}\left(w_{0}, v_{0}\right) \\
& \quad=\left(w^{n}\right)^{\prime}\left(\gamma^{\prime}\left(w^{n}\right)+J_{0}^{\prime}(0) \gamma\left(w^{n}\right)\right)+\int_{0}^{w^{n}} J_{0}^{\prime \prime} \beta\left[\left(w-W_{n}\right)^{2}-\left(V_{n}\right)^{2}\right]^{1 / 2} \gamma(w) d w .
\end{aligned}
$$

Since $J_{0}(u)$ is an even integral function and $\gamma(w) \varepsilon C^{\prime}$ at least, it is clear that $Y^{\prime \prime}\left(w_{0}, v_{0}\right) \varepsilon$ C.

We remark next that (1.0) and (1.07) have a unique solution. Indeed this amounts to the statement that (1.0) has only the trivial solution $z\left(w_{0}, v_{0}\right)=0$ if the data on $P$ are now either $z_{v}=0$ or $z=0$. These are essentially known results in the theory of the mixed problem for hyperbolic equations.

We have already shown that the solution $z\left(w_{0}, v_{0}\right)$ found as described in the previous paragraphs satisfies (1.0). We wish now to show that (1.07) is satisfied. It is easy to establish this for the data on $t=0$ and $x=l$. Hence we restrict ourselves to the data on $P$. Evidently the solution we have found satisfies (3.05). Suppose, however, it is inconsistent with the data on $P$. Let $s\left(w_{0}, v_{0}\right)$ be a solution of (1.0) and (1.07). Then $s(w, 0)$ satisfies (3.05). Write $y\left(w_{0}, v_{0}\right)=s\left(w_{0}, v_{0}\right)-z\left(w_{0}, v_{0}\right)$. Manifestly $y(w, 0)$ satisfies (3.05) with $\psi(w)$ replaced by 0 . However, this amounts to the statement that

$$
y(w, 0)=\int_{0}^{w} K(w, s) y(s, 0) d s
$$

where $K\left(w^{0}, s\right)$ is a finite sum of the bounded piecewise continuous functions $E\left(s, w^{0}\right)$ 
defined previously. The classical theory of the Volterra equation then guarantees that $y(w, 0)=0$ since the possible discontinuities of $E\left(s, w^{0}\right)$ are on $w^{0}-s=C$, i.e. lines inclined to the axes. Our uniqueness assertion of the preceding paragraph implies now that $y\left(w_{0}, v_{0}\right)=0$. Hence $z\left(w_{0}, v_{0}\right)$ satisfies the datum condition on $P$.

We carry through the validation of the solution of problem 2 in a somewhat more direct manner. It is plain from (6.08) that $g\left(w, v_{0}\right)$ has derivatives of all orders. Hence $\psi(w) \varepsilon C^{\prime}$ implies by (6.09) that $z\left(w_{0}, v_{0}\right) \varepsilon C^{2}$. Formal differentiation of the integral of type (5.0) with integrand $\exp \left(p w^{0}\right) H\left(p, v_{0}\right)$ indicates that this integral and hence $z\left(w_{0}, v_{0}\right)$ satisfies (1.0). This surmise is established rigorously by replacing $g\left(w_{0}, v_{0}\right)$ by any of the terms in (6.08) whence it appears that the right hand side of (6.09) satisfies (1.0). To show proper datum values are assumed on $P$ we observe first that the independent variables are $w_{0}, v_{0}$ and not $w^{0}, v_{0}$. We require now that $\psi(w) \varepsilon C^{2}$ and that the modified function satisfies: $d^{2} \psi(w) / d w^{2}$ is Lebesgue integrable on $0 \leq w<\infty$. Then $|D(p)| \leq M|p|^{-2}$. Hence with $H_{1}\left(p, v_{0}\right)=H\left(p, v_{0}\right) \exp \left(-p v_{0}\right)$

$$
\frac{\partial}{\partial v_{0}} H_{1}\left(p, v_{0}\right)
$$

can easily be shown to admit an inverse transform with kernel exp $w_{0} p$ and this is precisely $\partial z / \partial v_{0}$. There is no difficulty in taking the limit as $v_{0}$ goes to 0 . Indeed it will be clear that we have

$$
\begin{aligned}
\left|\int_{d-i \infty}^{d+i \infty}\left(\frac{\partial H_{1}}{\partial v_{0}}-\left.\frac{\partial H_{1}}{\partial v_{0}}\right|_{\nu_{0}-0}\right) e^{w^{\circ} p} d p\right| \\
\quad \leq M \int_{d-i A}^{d+i A}|p|^{-2}\left(\left|1-e^{-v_{0} \alpha}\right|+\mid 1-e^{\left(v_{0}-2 \lambda_{\rho} l\right) a}\right)\left|e^{\omega^{\infty} p}\right| d p
\end{aligned}
$$

for proper choice of $A$. For $v_{0}$ sufficiently small it is trivial that the right hand side is dominated by $2 \epsilon$. Hence in view of (6.02)

$$
\begin{aligned}
\left.\frac{\partial}{\partial v_{0}} H_{1}\left(p, v_{0}\right)\right|_{v_{0}=0} & =q D(p) S(p)\left(1-e^{-2 l \rho \lambda a}\right) \\
& =-D(p) S(p)\left[\left(h\left(1+e^{-2 l \rho \lambda a}\right)-(q+h)+(q-h) e^{-2 l \rho \lambda a}\right]\right. \\
& \sim h z\left(w^{0}, 0\right)+\psi\left(w^{0}\right)
\end{aligned}
$$

This establishes that the boundary values are assumed on $P$ when $\psi(w) \varepsilon C^{2}$. (To gain the result for the weaker hypothesis $\psi(w) \varepsilon C^{\prime}$ is not difficult though it seems undesirable to give more than a sketch of one procedure. Let $S_{1}$ be the segment of $P, 0 \leq$ $w \leq A$ and $S_{2}$ be the region bounded by $S_{1}, P^{\prime}, t=0$ and the minus characteristic through $(A, 0)$. Let $B_{1}$ be the Banach space of functions of class $C^{\prime}$ on $S_{1}$ which vanish together with their first derivatives ${ }^{11}$ at $w=0$. Let $B_{2}$ be the Banach space of functions of class $C^{2}$ over $S_{2}$. We are able to show that the transformation $T$ which maps $\psi(w)$ into $z(w, v)$ is linear on $B_{1}$ into $B_{2}$. The functions of Class $C^{2}$ on $S_{1}$ which vanish together with their first derivatives at $w=0$ are dense in $B_{1}$. Hence the linearity of $T$ ensures

${ }^{11} \mathrm{~S}$. Banach, Théorie des opérations linéaires, Warsaw, 1932. 
that the boundary conditions are satisfied on $P$ for $\psi(w) \varepsilon C^{\prime}$ since this is the fact for $\psi(w) \varepsilon C^{2}$. Of course, this result can also be established directly by the method followed in connection with problem 1 ).

The equation (1.0) has been basic in this paper. In many applications

$$
z_{t t}-z_{x x}-z=0
$$

occurs; that is to say the roles of $x$ and $t$ are interchanged. This implies that the Riemann function for (1.0)* is obtained from that for (1.0) by interchanging $x$ and $t$. This amounts to replacing $\beta$ by $i \beta$. We assert in fact that all the results of our paper may be interpreted for $(1.0)^{*}$ as the fundamental equation by just this substitution of $i \beta$ for $\beta$. This is plain for (3.03) and (3.05) since the Bessel's functions occurring there appear as the Riemann functions. We remark that $\beta$ enters the $p$ functions arising from (1.0)* in the combination $\left(p^{2}-\beta^{2}\right)^{1 / 2}$ instead of the previous $\left(p^{2}+\beta^{2}\right)^{1 / 2}$. A little reflection will show that our italicized assertion will be established if we show that the correspondence between $p$ functions and the inverse transforms is maintained when $\beta$ is replaced by $i \beta$. This is at once verified for (6.01). Write

$$
\begin{aligned}
q_{1} & =\left(p^{2}-\beta^{2}\right)^{1 / 2} \\
Q_{1}(u, B) & =\frac{1}{2 \pi i} \int_{d-i \infty}^{d+i \infty}\left[\left(e^{u p-B a_{1}}\right) /\left(q_{1}+h\right)^{n} q_{1}\right] d p .
\end{aligned}
$$

Then

$$
-\frac{\partial}{\partial B} Q_{1}(u, B)=\frac{1}{2 \pi i} \int_{d-i \infty}^{d+i \infty}\left(q_{1}+h\right)^{-n} e^{u p-B q_{1}} d p .
$$

Straightforward differentiation of $Q_{1}(u, B)$ reproduces the Eq. (5.08) with $i \beta$ replacing $\beta$ on the right hand side in view of our comment on (6.01) and $Q_{1}(u, B)$ replacing $Q(u, B)$. Accordingly (5.10), (5.11) and (5.12) are still valid when $Q_{1}(u, B)$ replaces $\boldsymbol{Q}(u, B)$ and $i \beta$ replaces $\beta$. Evidently all arguments based on the behavior of $q_{1}$ as $|\boldsymbol{p}| \rightarrow$ $\infty$ are identical with those involving $q$. 